\title{
NEWMEDS special issue commentary
}

\author{
Tine Bryan Stensbøl ${ }^{1}$ - Shitij Kapur ${ }^{2}$
}

Published online: 26 September 2015

(C) Springer-Verlag Berlin Heidelberg 2015

\section{Introduction}

The Innovative Medicines Initiative (IMI) is Europe's largest public-private initiative aiming to speed up the development of better and safer medicines for patients - and nowhere is the need more urgent than in the area of CNS medications (2015a). IMI is a joint undertaking between the European Union and the pharmaceutical industry association, European Federation of Pharmaceutical Industries and Associations (EFPIA), and supports collaborative research projects between the academia and industry. NEWMEDS (Novel Methods leading to New Medications in Depression and Schizophrenia) which generated the articles in this supplement is one of the earliest IMI (2015b). In this Commentary, we introduce the Innovative Medicine Initiative and what makes it unique; we provide perspective on some of the achievements of NEWMEDS and some lessons learned in the process of this partnership over 5 years.

The way the IMI consortia are brought together is unique. Before the Call is launched, a consortium of EFPIA companies and, in some cases, other large companies or organizations active in health research agree on the need to work together and with other stakeholders on a specific issue. The topic text is drafted and, following consultation with various groups, the details of the Call text is sent to the IMI Governing Board for approval.

Shitij Kapur

Shitij.kapur@kcl.ac.uk

$1 \quad$ H. Lundbeck A/S, Valby, Denmark

2 Institute of Psychiatry, Psychology and Neuroscience, King's College London, London, UK
Stage 1 Once the Governing Board has given its green light, the Call is published and all interested parties from academia, small- and medium-sized enterprises (SMEs), patient organizations, regulatory agencies, etc. are invited to form consortia and to submit a proposal in response to the Call. The proposals are screened for eligibility and reviewed by independent experts. The experts rank the applications, and the consortium that submitted the top-ranked application is invited to proceed to Stage 2 .

Stage 2 In stage 2, the top-ranked applicant consortium and the EFPIA consortium behind the topic are invited to form a full consortium and submit a full proposal. The full proposal is also reviewed by independent experts. The proposal that get approval at both stages, and where the industrial partners are willing to match the public funds $1: 1$, get to proceed.

\section{NEWMEDS-a new way of collaboration}

NEWMEDS is one such project - a consortium of 23 participating institutions based in nine different EU member states as well as in Iceland, Switzerland and Israel —with Lundbeck as the main pharmaceutical coordinator, King's College London as the academic coordinator, and SME GABO:mi (Munich) taking on the administrative project management tasks. The stage 1 of NEWMEDS was initiated in 20082009 , and it was designed to (a) develop standardized animal models that focus on reliable cross-species endophenotypes (e.g. cognitive function) and use cross-species methods (MRI, EEG) to bring animal models closer to clinical endpoints; (b) develop fMRI- and PET-based paradigms which may serve as early or surrogate markers to provide guidance for drug development; (c) examine a set of genetic abnormalities (copy-number variation (CNV)) closely linked with 
schizophrenia by studying them in human populations and animal models to achieve one-to-one cross-species correlation of the impact of these mutations on cognition, behaviour and brain physiology and function; (d) identify pharmacogenetic biomarkers that can be used to stratify patients within an umbrella DSM diagnosis (e.g. depression) thus allowing for targeted clinical trials and individualized treatment; and (e) provide new insights for the intelligent design of clinical trials.

Articles in this issue of the journal address the issue of standardization of animal model application and their translation into early experimental trials in humans. Two issues become immediately evident: the complexity of measurement and modelling issues in animal models is daunting and the advances in relating animal models to human outcomes are reassuring.

\section{Delivering treatments for cognition-a quest for standardization, translation and validation}

The special issue carries 27 articles emerging from the NEWMEDS collaboration. This is a small segment of the overall activity capture by the overall strategy. However, the articles collectively exemplify the three major challenges that bedevil the process of "identifying and validating" a target and then translating it into a therapeutic possibility.

Hvoslef-Eide et al. (2015) and Lees et al. (2015) demonstrate two ends to the quest for standardization. In this regard, NEWMEDS has made important contributions. The NEWMEDS consortium has worked to develop standardized methods for evaluating cognition in rodents and humans, respectively. Perhaps, the most striking about the effort has been the industrial collaboration-where experiments have been repeated across competitor companies using the same methods to enhance reliability and standardization (Young et al. 2015)

Standardization of animal models alone does not make them valid. And this remains an ongoing challenge. Studies across the different models currently in use, eg. subchronicPCP, MK801 and ketamine, all show abnormalities that could be related to some aspects of the many manifestations of schizophrenia - but, none that is so convincingly superior to the others. As a result, all of them remain partially validadding complexity to the drug hunter's quest. While not detailed in this issue, perhaps the only way to validate these models would be against well-implicated genetic causes. In that regard, work reported by NEWMEDS elsewhere using the major CNVs implicated in schizophrenia may show new pathways to identification of novel targets and subsequent validation (Stefansson et al. 2014).

But, standard and valid animal models are of limited utility unless their lessons can be translated into experimental human studies. Here, the progress in the tools of translation has been encouraging. Gass and colleagues (2015) show how resting- state fMRI can be used in Sprague-Dawley rats and healthy human volunteers to study the effect of ketamine with similar results, and Joules and colleagues (2015) show how one can use advanced machine learning methods to distinguish the effects of different drugs on a ketamine model.

\section{Lessons from NEWMEDS academic-industry collaborations}

NEWMEDS was one of the first projects under the IMI initiative, and it brought together expert scientists in the field of psychiatry from the industry as well as leading academic institutions around Europe. This was a new experience for all partners - especially in terms of scope and scale. What did it teach us about such collaborations?

First, we realized that professional project management was critical to success. NEWMEDS was a multi-industry, multi-academic, geographically diffuse partnership - it was held together and delivered due to dedicated project management that was there right from the beginning. Second, we found that a scientific focus was critical to have but hard to maintain. Balancing the "depth" that science demands and "breadth" that partnerships demand is a constant tension that needed to be balanced. Keeping all partners on board required a constant juggling of this balance. Third, we realized that despite the ubiquity of emails and social media, it was still necessary to meet and talk. We found the following mechanisms indispensable: (a) a biweekly teleconference of the trio of industry lead/academic lead/project management; (b) a quarterly written follow-up on milestones and deliverables by all the Workpackages (WP); (c) six monthly teleconferences between the "trio" and the WP leads based on the quarterly updates; and (d) yearly face-to-face meetings with the whole consortium - updating on the scientific advances across the packages.

Finally, we observed that while these projects are about scientific questions, the outcomes hinge upon the unique interests and expertise of individual scientists in the industry and academia. We were struck by the asymmetrical change in turnover in academic and industry leads. Through the project, all the academic leads remained, while three industrial partners exited schizophrenia and depression and four changed their lead scientists. This was a challenge. Perhaps nothing can be done about it — but those embarking on future ventures would do well to be aware of this reality.

We are often asked "so when will the new medications in psychiatry come?" We wish we could answer that with certainty. The process of drug development in CNS still remains unpredictable and takes long. However, the tools that NEWMEDS have developed, and the advances outlined here and elsewhere, make a meaningful contribution towards making this journey a bit faster and a bit more predictable. And above all, the project demonstrates how competing private companies and academic labs can collaborate towards a larger goal. 
Competing interest SK has received a recent grant support from Lundbeck and Roche, has served as a one-off consultant and/or speaker for Otsuka, Sunovion and Takeda and served on the Scientific Advisory Boards for Acadia, Lundbeck and Roche.

\section{References}

(2015a) Innovative Medicines Initiative http://www.imi.europa.eu/, pp IMI Website

(2015b) NEWMEDS Consortium http://www.newmeds-europe.com/

Gass N et al (2015) Acute ketamine challenge increases resting state prefrontal-hippocampal connectivity in both humans and rats. Psychopharmacology this issue.

Hvoslef-Eide M, Mar A, Nilsson S et al (2015) The NEWMEDS rodent touchscreen test battery for cognition relevant to schizophrenia: psychopharmacology this issue.
Joules R, Doyle OM, Schwarz AJ et al (2015) Ketamine induces a robust whole-brain connectivity pattern that can be differentially modulated by drugs of different mechanism and clinical profile. Psychopharmacology (this issue)

Lees J, Applegate E, Emsley E, et al (2015) Calibration and crossvalidation of MCCB and CogState in schizophrenia: psychopharmacology this issue.

Stefansson H, Meyer-Lindenberg A, Steinberg S, Magnusdottir B, Morgen K, Arnarsdottir S, Bjornsdottir G, Walters GB, Jonsdottir GA, Doyle OM, Tost H, Grimm O, Kristjansdottir S, Snorrason H, Davidsdottir SR, Gudmundsson LJ, Jonsson GF, Stefansdottir B, Helgadottir I, Haraldsson M, Jonsdottir B, Thygesen JH, Schwarz AJ, Didriksen M, Stensbol TB, Brammer M, Kapur S, Halldorsson JG, Hreidarsson S, Saemundsen E, Sigurdsson E, Stefansson K (2014) CNVs conferring risk of autism or schizophrenia affect cognition in controls. Nature 505:361-366

Young D, Mohler E, Ding Z et al (2015) Cross-site strain comparison of pharmacological deficits in the touchscreen visual discrimination test. Psychopharmacology this issue 